\title{
UN MÉTODO PARA ENSEÑAR EL PORQUÉ SUCEDEN LAS REACCIONES QUÍMICAS
}

\author{
Victor A. López-Agudelo, Daniel Barragán* y Wilson Parra \\ Escuela de Química, Facultad de Ciencias, Universidad Nacional de Colombia, Calle 59A № 63-20, Bloque 16, Núcleo El Volador, \\ Medellín, Colombia
}

Recebido em 12/4/12; aceito em 26/8/12; publicado na web em 29/11/12

\begin{abstract}
AN APPROACH FOR EXPLAINING CHEMICAL TRANSFORMATIONS. A methodology is proposed for explaining one of the central questions in the teaching of general chemistry courses to freshman students: why do chemical transformations occur? The answer to this question is based on thermodynamics but we propose arriving at an answer in a more intuitive way by using computational tools in a bid to increase the motivation of students for learning chemistry.
\end{abstract}

Keywords: molecular modeling; energy minimization; reactivity.

\section{INTRODUCCIÓN}

Es común encontrar que en los cursos básicos de química el tema de la reactividad de las sustancias químicas se presente de manera cualitativa, usualmente como una clasificación de las reacciones agrupadas por características comunes, tales como la transferencia de electrones entre moléculas, la sustitución o el dezplamiento de átomos de una molécula y la combinación o descomposición de moléculas. ${ }^{1}$ Si bien esta primera aproximación al concepto de reactividad ayuda a que los estudiantes reconozcan que las sustancias que participan de una reacción química deben tener unas propiedades específicas y que hay ciertos requerimientos energéticos involucrados, también puede dar la impresión de que no hay una clara conexión con los temas de enlace químico y geometría molecular. Con el propósito de disminuir la percepción de abstracción en el aprendizaje de la química por parte de los estudiantes, es conveniente desarrollar estrategias metodológicas que muestren que es posible abordar los temas de la química de una manera coherente. En este sentido encontramos conveniente que al presentar la reactividad química de la sustancias, se haga una clara distinción entre el cómo se lleva a cabo la reacción y el porqué la reacción sucede. ${ }^{2,3}$ El cómo sucede la reacción es una pregunta cercanamente relacionada con los mecanismos propuestos para explicar la redistribución de los electrones en la molécula después de la ruptura y formación de enlaces químicos, mientras que el porqué sucede la reacción es una pregunta que debe ser entendida con la direccionalidad y espontaneidad del proceso y que tiene respuesta precisa en termodinámica: las reacciones químicas tienen lugar en la dirección en la que disminuye la energía de Gibbs del sistema, a temperatura y presión constantes, independientemente del signo que acompaña los cambios de entalpía y de entropía. Desafortunadamente introducir algunos conceptos de la termodinámica en el inicio del aprendizaje de la química puede provocar que aumente en el estudiante la percepción de complejidad para entender la química. ${ }^{4,5}$

El modelamiento molecular es una herramienta que ofrece la oportunidad de innovar continuamente en los procesos pedagógicos de enseñanza de la química, dando la posibilidad de promover en el estudiante el interés y el entendimiento de la química a la vez que desarrolla en él habilidades y destrezas que potencializan sus capacidades cognitivas. ${ }^{6,7}$

En este artículo presentamos una metodología que tiene como proposito ayudar a que los estudiantes comprendan el porqué se lleva

*e-mail: dalbarraganr@unal.edu.co a cabo una reacción química, sin necesidad de recurrir explicitamente a la termodinámica y haciendo uso de los fundamentos de la física que nos ayudan en primera instancia a explicar la estructura y geometría molecular: la estructura química más estable de una determinada molécula es la que tiene la mínima energía potencial, entre todas las posibles configuraciones de sus átomos en el espacio. ${ }^{8}$ En esencia necesitamos lo siguiente conceptos: de la física clásica los de fuerza de repulsion-atracción electrostática y energía potencial eléctrica, de las primeras lecciones de química el de geometría molecular a través de la teoría VSEPR y algunos mecanismos sencillos para explicar la ruptura y formación de enlaces químicos y finalmente una herramienta computacional que nos permita optimizar la geometría molecular y modelar una reacción.

De los fundamentos de física es suficiente con recordarles a los estudiantes que la magnitud de la fuerza eléctrica, ya sea de atracción o repulsión, entre partículas con carga varia proporcionalmente con la magnitud de la carga e inversamente con el cuadrado de la distancia entre partículas, y que la energía potencial eléctrica en sistemas de partículas con carga eléctrica depende proporcionalmente de la masa y carga de las particulas e inversamente con la distancia. ${ }^{9,10} \mathrm{Con}$ estos conceptos básicos esperamos que los estudiantes de química puedan visualizar que la energía potencial eléctrica de un sistema de particulas con carga depende de la configuración espacial del sistema, es decir de la posición relativa de las particulas en el espacio. Por otra parte en los libros de química general encontramos que la teoría VSEPR (valence-shell electron-pair repulsion) se utiliza para dar una primera explicación de la geometría de las moléculas. ${ }^{10,11}$ La teoría $V S E P R$ postula que una molécula alcanza una geometría especial que minimiza la repulsión eléctrica entre los electrones de la capa de valencia. ${ }^{1,8}$ Ahora, si ponemos juntos los fundamentos de la física de partículas con carga y la teoría VSEPR, esperamos que el estudiante pueda visualizar lo que sucederá con la energía de una molécula al adicionar o quitar átomos mediante una reacción química, y esto es que la energía cambiará porque los átomos de la molécula tendrán que reorientarse espacialmente y alcanzar una nueva geometría que minimice la repulsiones eléctricas entre los electrones de la capa de valencia de los átomos. ${ }^{12}$

El conocimiento previo de los estudiantes es el punto de partida para construir nuevo conocimiento a través de la metodología que presentamos. ${ }^{13}$ Los estudiantes deben escoger una reacción que quieran estudiar, deben proponer como se redistribuirán los electrones en las moléculas después de la ruptura y formación de enlaces químicos a través de un estado intermedio de transición y luego usando la herramienta 
computacional deben calcular como varía la energía potencial del sistema a medida que se forma o se rompe un enlace químico. ${ }^{14} \mathrm{Si}$ el perfil de energía potencial del sistema de reacción muestra un mínimo en función de la coordenada de reacción o restricción de enlace, que es el enlace elegido para seguir el curso de la reacción, entonces el mecanismo propuesto es posible y la reacción tendrá lugar. ${ }^{15}$

\section{PARTE EXPERIMENTAL}

La teoría VSEPR no proporciona ningún tipo de información relacionada con la energía de las moléculas, sinembargo esta información se puede obtener a partir de las coordenadas espaciales de los átomos de la molécula, de las distancias y ángulos de enlace a través de los métodos de la química computacional que optimizan la geometría molecular. ${ }^{8,15}$ El estudio computacional de cualquier reacción química tiene como uno de sus objetivos determinar la correlación que hay entre la energía de las estructuras moleculares de equilibrio de los reactivos y de los productos con la estructura transitoria propuesta para explicar la redistribución de los electrones después de la ruptura y formación de enlaces químicos. Esta correlación usualmente se aprecia en una superficie de energía potencial, en la cual los mínimos de energía para reactivos y productos están conectados a través de un máximo correspondiente al estado molecular transitorio que se ha propuesto. Los métodos semi-empíricos de la química computacional permiten optimizar la geometría de un amplio conjunto de moléculas y sistemas moleculares de una manera rápida y exacta, ya que utilizan información experimental de las moléculas y realizan un gran número de aproximaciones durante los cálculos de optimización. Entre estos métodos el AM1 es ampliamente conocido y viene incorporado en la gran mayoría de programas de uso en química computacional. ${ }^{15}$ En este trabajo utilizamos el programa Spartan de Wavefunction Inc. y el método semi-empírico AM1 para optimizar la geometría de las moléculas estudiadas y para calcular los perfiles de energía potencial de los estados transitorios propuestos para las diferentes reacciones utilizadas para ilustrar la metodología propuesta. ${ }^{16}$

\section{RESULTADOS Y DISCUSION}

Para llevar a cabo este trabajo seleccionamos un conjunto de 8 reacciones que son representativas de los ejemplos que usualmente encontramos en libros de texto de química general y química orgánica, y las cuales podemos agrupar en dos series de reactividad, alifáticas y aromáticas. ${ }^{1,14}$ En la Tabla 1 mostramos los valores de energía libre estándar de las 8 reacciones, calculados a partir de la optimización de la geometría molecular con el método semi-empírico AM1. Los valores negativos e inclusive el valor positivo obtenido para la energía libre estándar en la Tabla 1 indican que las reacciones ocurren preferiblemente en la dirección que favorece la formación de productos, y que por lo tanto sirven de soporte a los resultados que se mostraran más adelante. Todas estas reacciones son ampliamente conocidas y estudiadas experimentalmente en química orgánica y por tanto para cada una de ellas se conocen otra serie de condiciones que se deben tener en cuenta para favorecer el rendimiento de la reacción, tales como temperatura, $\mathrm{pH}$, solventes y catalizadores. ${ }^{14}$ En la Tabla 1 también mostramos, para cada reacción, la restricción de enlace o coordenada interna de reacción seleccionada para seguir la variación en la energía potencial del sistema transitorio propuesto a medida que la reacción sucede, esta coordenada corresponde a la longitud del enlace químico que se está formando. ${ }^{14,17}$

Como ya se mencionó, las 8 reacciones de la Tabla 1 se agrupan en dos series de reactividad química, sustituciones nucleofílicas alifáticas $\left(\mathrm{SN}_{2}\right)$ y sustituciones electrofílicas y nucleofílicas aromáticas. En la Figura 1 mostramos la primera serie de reactividad para
Tabla 1. Energía libre estándar y restricción de enlace o coordenada interna de reacción para el conjunto de reacciones seleccionadas. Los cálculos se realizaron con el método semi-empírico AM1

\begin{tabular}{lcc}
\hline Reacción química & $\begin{array}{c}\text { Coordenada } \\
\text { de reacción }\end{array}$ & $\begin{array}{c}\Delta \mathrm{G}^{\circ} \\
\left(\mathrm{kJ} \mathrm{mol}^{-1}\right)\end{array}$ \\
\hline (a) $\mathrm{CH}_{3} \mathrm{SCN}+\mathrm{OH}^{-} \rightarrow \mathrm{CH}_{3} \mathrm{OH}+\mathrm{SCN}^{-}$ & $\mathrm{C}-\mathrm{O}$ & $-319,79$ \\
(b) $\mathrm{CH}_{3} \mathrm{Cl}+\mathrm{OH}^{-} \rightarrow \mathrm{CH}_{3} \mathrm{OH}+\mathrm{Cl}^{-}$ & $\mathrm{C}-\mathrm{O}$ & $-174,38$ \\
(c) $\mathrm{CH}_{3} \mathrm{I}+\mathrm{Cl}^{-} \rightarrow \mathrm{CH}_{3} \mathrm{Cl}+I^{-}$ & $\mathrm{C}-\mathrm{Cl}$ & $-15,71$ \\
(d) $\mathrm{CH}_{3} \mathrm{SCN}+\mathrm{Cl}^{-} \rightarrow \mathrm{CH}_{3} \mathrm{Cl}+\mathrm{SCN}^{-}$ & $\mathrm{C}-\mathrm{Cl}$ & $-145,41$ \\
(e) $\mathrm{CH}_{3} \mathrm{SCN}+\mathrm{Br} \rightarrow \mathrm{CH}_{3} \mathrm{Br}+\mathrm{SCN}^{-}$ & $\mathrm{C}-\mathrm{Br}$ & $-125,78$ \\
(f) $\mathrm{C}_{6} \mathrm{H}_{6}+\mathrm{CH}_{3} \mathrm{Cl} \rightarrow \mathrm{C}_{6} \mathrm{H}_{5} \mathrm{CH}+\mathrm{HCl}$ & $\mathrm{C}-\mathrm{C}$ & $-46,79$ \\
(g) $\mathrm{C}_{6} \mathrm{H}_{6}+\mathrm{Cl}_{2} \rightarrow \mathrm{C}_{6} \mathrm{H}_{5} \mathrm{Cl}+\mathrm{HCl}$ & $\mathrm{C}-\mathrm{Cl}$ & $-87,3$ \\
(h) $\mathrm{C}_{6} \mathrm{H}_{5} \mathrm{Cl}+\mathrm{H}_{2} \mathrm{O} \rightarrow \mathrm{C}_{6} \mathrm{H}_{5} \mathrm{OH}+\mathrm{HCl}$ & $\mathrm{C}-\mathrm{O}$ & $+1,81$ \\
\hline
\end{tabular}

las sustituciones nucleofílicas alifáticas, donde los valores en el eje de las abscisas corresponde al mínimo de energía potencial que optimiza la geometría molecular de la respectiva molécula alifática, calculado mediante el método AM1. A esta Figura 1 la denominamos serie de reactividad porque le facilita al estudiante visualizar cuales sustituciones nucleofílicas sobre las moléculas dan lugar a reacciones que suceden de manera espontánea, igualmente permite visualizar claramente cuáles moléculas son más reactivas que otras al comparar la magnitud de la energía molecular, por ejemplo, en esta serie el metanol es la sustancia menos reactiva de la serie.

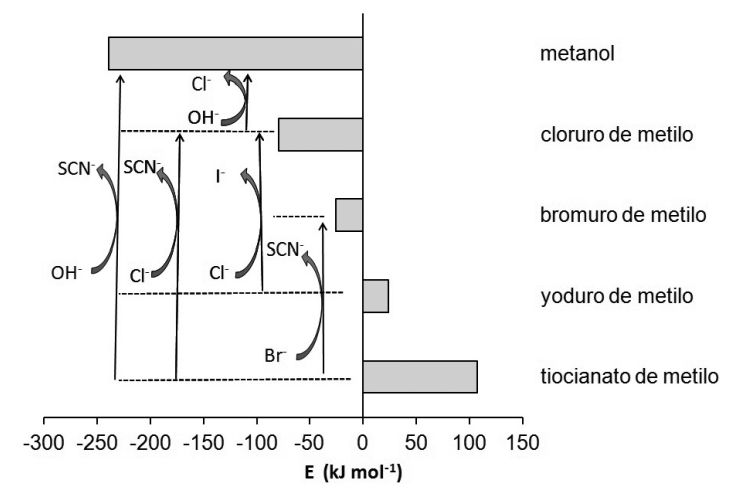

Figura 1. Serie de reactividad alifática para sustituciones nucleofílicas, $\mathrm{SN}_{2}$. El valor de energía en el eje de las abscisas corresponde al mínimo de energía en la optimización de la geometría molecular AM1 y las barras horizontales indican la respectiva molécula. Las flechas dentro de la gráfica indican la sustitución nucleofílica para que suceda la reacción, según los datos de la Tabla 1

En la Figura 2 mostramos a modo de ejemplo una de las reacciones de la Figura 1. El tiocianato de metilo es más reactivo que el bromuro de metilo, por eso es posible sustituir el grupo tiocianato por el bromuro. El mecanismo de reacción mediante el cual se lleva a cabo esta reacción requiere de un análisis químico más avanzado, ${ }^{14}$ sin embargo es de esperar que de la Figura 1 los estudiantes puedan visualizar que durante la ruptura y formación del nuevo enlace químico, el grupo tiocianato sale con los dos electrones del enlace mientras que el bromuro aporta los dos electrones que requiere el enlace que se forma, este es el significado de las flechas en la Figura 2. ${ }^{1}$

Una vez se ha propuesto el estado transitorio para cada una de las reacciones, como el ilustrado en la Figura 2, se procede a calcular el perfil de energía potencial del sistema en función de la restricción o coordena interna de reacción seleccionada, esto se hace a través de la simulación aproximando a la molécula el grupo entrante y alejando 


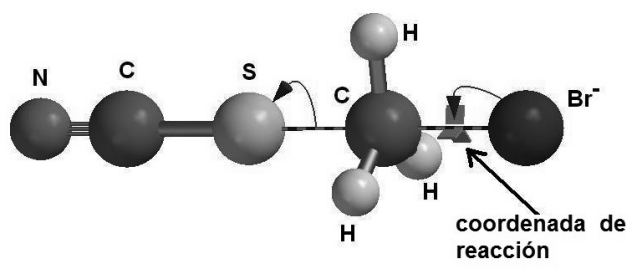

Figura 2. Estructura transitoria propuesta para modelar la reacción entre el Bry el $\mathrm{CH}_{3} \mathrm{SCN}$. Las flechas indican como se distribuyen los electrones durante la reacción. La restricción de enlace o coordenada interna de reacción (bond constraint) es la distancia que disminuye durante el cálculo de la energía potencial para el ataque nucleofilico bimolecular

el grupo saliente..$^{14,15}$ En la Figura 3e se muestra el perfil de energía obtenido para la reacción de la Figura 2. Inicialmente el ion bromuro se encuentra a una distancia de $2.2 \AA$ del tiocinato de metilo, se observa que a medida que esta distancia se acorta, la energía potencial del sistema molecular transitorio disminuye a medida que el grupo tiocinato se aleja de la molécula, alcanzando un mínimo de energía a una distancia de $1.93 \AA$; este es el valor aceptado para la longitud del enlace Br-C. ${ }^{1,14}$ Es importante hacer notar que el valor de energía en el mínimo de la Figura 3e no coincide con el valor de energía para el bromuro de metilo en la Figura 1, ya que se esta calculando para todo el sistema molecular transitorio propuesto en la Figura 2.

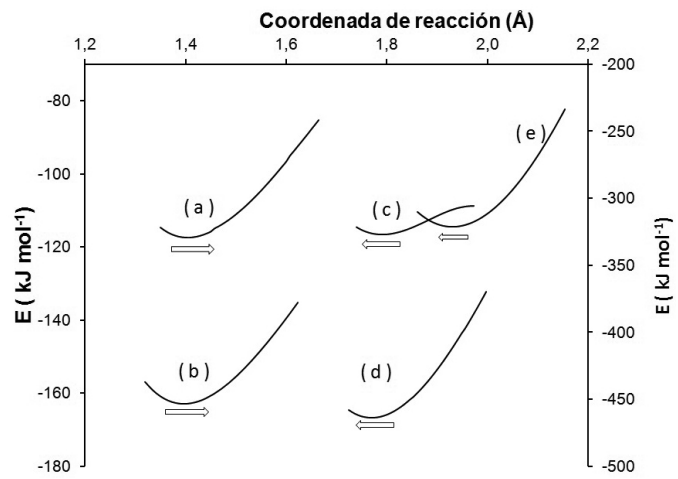

Figura 3. Perfiles de energía potencial en función de la respectiva restricción de enlace para la serie de reactividad de la Figura 2: (a) $\mathrm{OH}^{-}+\mathrm{CH}_{3} \mathrm{SCN}$; (b) $\mathrm{OH}^{-}+\mathrm{CH}_{3} \mathrm{Cl}$; (c) $\mathrm{Cl}^{-}+\mathrm{CH}_{3} \mathrm{I}$; (d) $\mathrm{Cl}^{-}+\mathrm{CH}_{3} \mathrm{SCN}$; (e) $\mathrm{Br}^{-}+\mathrm{CH}_{3} \mathrm{SCN}$. Las flechas debajo de las curvas señalan el correspondiente eje de las ordenadas

En la Figura 3 se muestran los perfiles de energía de reacción en función de la restricción de enlace, Tabla 1, para cada una de las reacciones de la serie de reactividad de la Figura 2. Todos los mínimos de energía potencial están de acuerdo con los valores reportados para la longitud del respectivo enlace químico formado. ${ }^{14,17,18}$

En la Figura 4 se muestra la segunda serie de reactividad, que corresponde a reacciones aromáticas de sustitución electrofílica y nucleofílica. En esta serie los estudiantes podrán observar que el benceno tiene la energía de molécula más alta, lo que lo hace el más reactivo en la serie.

Para proponer un estado transitorio para alguna de las reacciones de la Figura 4 se requiere que los estudiantes tengan un poco más de intuición o práctica en proponer como se llevara a cabo la redistribución de electrones después de la ruptura y formación de los enlaces químicos. En la Figura 5 se da como ejemplo la estructura molecular transitoria propuesta para obtener clorobenceno a partir de benceno y cloro molecular. La cloración del benceno requiere de un catalizador y condiciones experimentales adecuadas y sucede via formación de un electrófilo promovido por las repulsiones electrostáticas entre el cloro molecular y el anillo aromático, de esta manera el enlace del

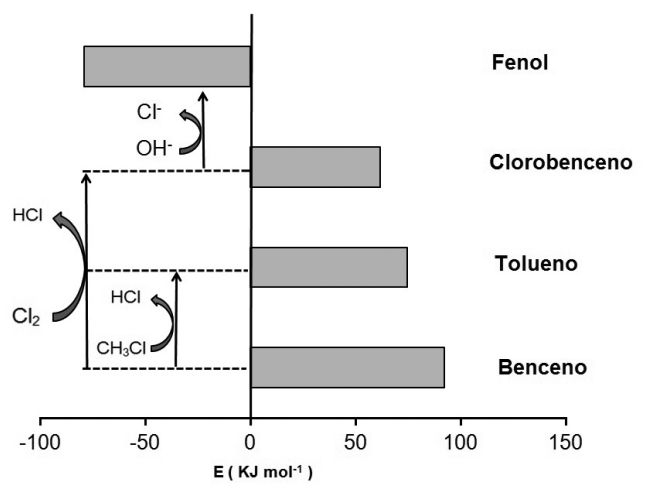

Figura 4. Serie de reactividad aromática para algunas sustituciones electrofílicas y aromáticas. El valor de energía en el eje de las abscisas corresponde al mínimo de energía en la optimización de la geometría molecular AM1 y las barras horizontales indican la respectiva molécula. Las flechas dentro de la gráfica indican la sustitución a realizar para que suceda la reacción, según los datos de la Tabla 1

cloro se rompe y el electrófilo ataca el anillo aromático desplazando un átomo de hidrógeno. ${ }^{14}$

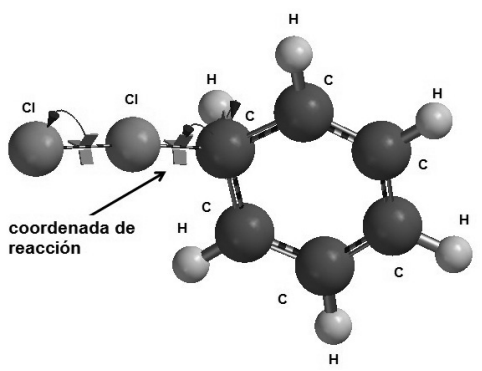

Figura 5. Estructura transitoria propuesta para modelar la reacción entre el cloro molecular y el benceno. Las flechas indican como se distribuyen los electrones durante la reacción. La restricción de enlace o coordenada interna de reacción (bond constraint) es la distancia que disminuye durante el cálculo de la energía potencial para la reacción bimolecular

En la Figura 6b se muestra el perfil de energía potencial del sistema molecular transitorio propuesto en la Figura 5, en función de la restricción de enlace seleccionada, Tabla 1. El mínimo de energía observado en la figura corresponde a la distancia del enlace químico Cl-C. ${ }^{17}$ Las otras curvas en la Figura 6 corresponde a los otros perfiles de energía potencial para las otras reacciones de la serie en la Figura 4.

Son varios los aspectos interesantes que se pueden destacar de este tipo de actividades donde el estudiante tiene la oportunidad de hacer experimentos de química en un computador, entre ellos que el estudiante puede probar todas sus ideas hasta obtener un resultado que sea significativo, disminuyendo así la sensación de frustración que tal vez podría llegar a sentir en un experimento fallido en el laboratorio. ${ }^{19}$ En la Figura 7a mostramos los perfiles de energía potencial en función de la distancia del enlace que se está formando para la reacción (b) en la Tabla 1, cuando el cálculo se realiza intercambiando el grupo entrante y el grupo saliente de la molécula. La curva (i) de la Figura 7a muestra como la energía potencial del sistema molecular transitorio propuesto siempre aumenta a medida que el cloruro se aproxima al metanol, haciendo el sistema más inestable, contrario a lo observado en la curva (ii) cuando el hidroxilo es quien se aproxima al cloruro de metilo. La herramienta computacional que utilizamos para calcular los perfiles de energía potencial de las reacciones, también da la posibilidad de obtener una gran variedad de información del sistema, como es la variación de las cargas electrostáticas sobre cada uno de los átomos a 


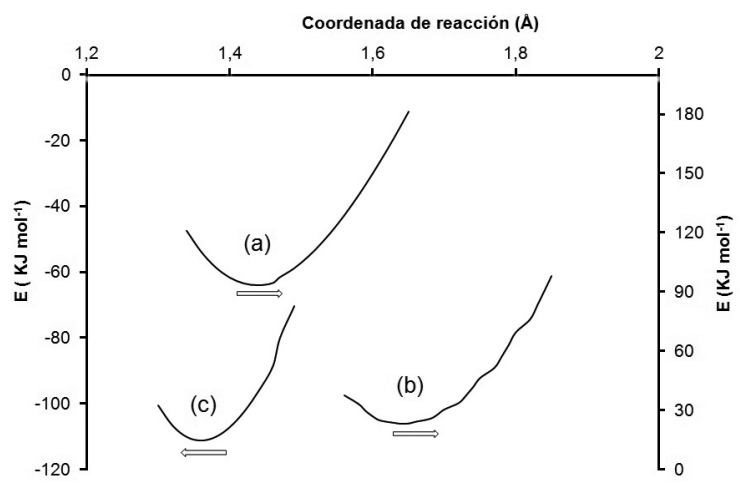

Figura 6. Perfiles de energía potencial en función de la respectiva restricción de enlace para la serie de reactividad de la Figura 4: (a) alquilación del benceno; (b) cloración del benceno; (c) hidroxilación del benceno

medida que la reacción transcurre. En la Figura 7b se muestra como varía la carga electrostática sobre los principales átomos involucrados en la reacción (b) de la Tabla 1 (Figura 1 y curva (ii) en Figura 7a). En la curva (ii) se aprecia como la carga sobre el átomo de cloro se aproxima a -1 a medida que el hidroxilo se aproxima al metilo, indicando que el cloro se está alejando de la molécula como ion cloruro. Las curvas (i) y (iii) muestran el comportamiento de la carga sobre los átomos de carbono y oxígeno, de una manera consistente a lo que está pasando durante la reacción. Estos resultados adicionales mostrados en la Figura 7 le ayudaran al estudiante a comprender con mayor claridad, no sólo porque sucede cada una de las reacciones sino como están sucediendo,

(a)
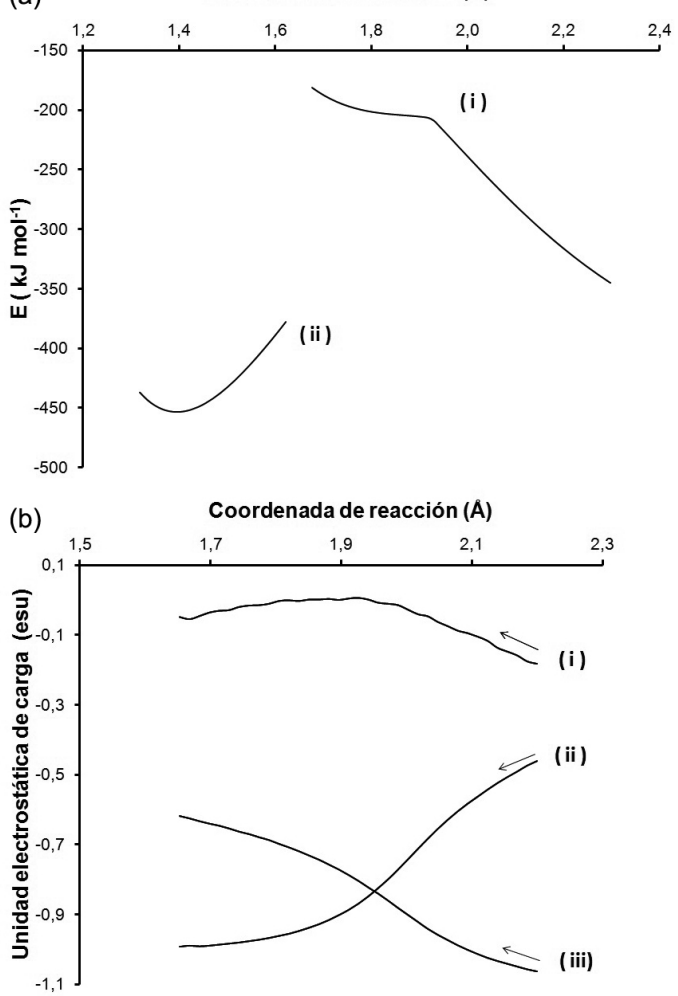

Figura 7. (a) Comparación de los perfiles de energía potencial para una reacción no espontánea y para una espontánea, al intercambiar grupo entrante y grupo saliente de la molécula: (i) $\mathrm{CH}_{3} \mathrm{OH}+\mathrm{Cl}^{-} \rightarrow \mathrm{CH}_{3} \mathrm{Cl}+\mathrm{OH}^{-}$; (ii) $\mathrm{CH}_{3} \mathrm{Cl}+\mathrm{OH}^{-} \rightarrow \mathrm{CH}_{3} \mathrm{OH}+\mathrm{Cl}^{-}$. (b) Variación de la carga electrostática sobre los átomos involucrados directamente en la ruptura y formación del enlace químico para la reacción $\mathrm{CH}_{3} \mathrm{Cl}+\mathrm{OH}^{-} \rightarrow \mathrm{CH}_{3} \mathrm{OH}+\mathrm{Cl}^{-}$: (i) átomo de $C$, (ii) átomo de $\mathrm{Cl}$, (iii) átomo $\mathrm{O}$ haciendo uso significativo de los conceptos previos de periodicidad química, enlace químico y geometría molecular. ${ }^{10}$

\section{CONCLUSIONES}

Los docentes siempre estamos en la continua búsqueda de estrategias que motiven a los estudiantes a interesarse por estudiar y aprender química, y los computadores nos ofrecen la oportunidad de contar con un laboratorio virtual para desarrollar la creatividad y nuevas ideas en el complejo proceso de enseñanza-aprendizaje de las ciencias. ${ }^{19-22}$ En este trabajo tomamos una herramienta computacional de un software comercial, la cual también se encuentra disponible en la mayoría de paquetes computacionales que no requiren de licencia para su uso, para utilizarla de manera contextualizada en la enseñanza del concepto de reactividad química en los cursos básico de química. La propuesta la llamamos una metodología, ya que se puede implementar para una clase magistral o para una sesión de laboratorio, desde una perspectiva en la cual se busca aclarar con argumentos sencillos la diferencia entre el cómo y el porqué suceden las reacciones químicas.

\section{AGRADECIMIENTOS}

A la División de Investigaciones - DIME of Universidad Nacional de Colombia - Medellín por el apoyo brindado para la realización del presente trabajo. Igualmente agradecemos a la profesora I. C. Perilla del Departamento de Química, Facultad de Ciencias, Universidad Nacional de Colombia - Bogotá, por lectura del manuscrito y los valiosos comentarios realizados.

\section{REFERENCIAS}

1. Brown, T. E.; LeMay, H. E.; Burste, B. E.; Murphy, C.; Woodward, P.; Chemistry: The Central Science, 12 ${ }^{\text {th }}$ ed.; Prentice Hall: New York, 2011.

2. Campbell, J. A.; Why Do Chemical Reactions Occur? Prentice-Hall: Englewood Cliffs, 1965.

3. Keeler, J.; Wothers, P.; Why Chemical Reactions Happen, Oxford University Press: New York, 2003.

4. Le Maréchal, J. F.; El Bilani, R.; Int. J. Thermodyn. 2008, 11, 91.

5. Sözbilir, M.; Pınarbaşı, T.; Canpolat, N.; Eurasia J. Math. Sci. Tech. 2010, 6, 111.

6. Ferreira, C.; Arroio, A.; Rezende, D.; Quim. Nova 2011, 34, 1661.

7. Khan, S.; J. Sci. Educ. Technol. 2011, 20, 215.

8. Gillespie, R. J.; J. Chem. Educ. 2004, 81, 298.

9. Halliday, D.; Resnick, R.; Krane, K. S.; Physics, $5^{\text {th }}$ ed.; John Wiley: New York, 2002, vol. 2.

10. Hurst, M. O.; J. Chem. Educ. 2002, 79, 763.

11. Lindmark, A. F.; J. Chem. Educ. 2010, 87, 487.

12. McNaught, I. J.; J. Chem. Educ. 2011, 88, 421.

13. Grove, N. P.; Bretz, S. L.; Chem. Educ. Res. Pract. 2012, 13, 201.

14. McMurry, J. E.; Organic Chemistry, $7^{\text {th }}$ ed., Thomson: USA, 2007.

15. Cramer, C. J.; Essential of Computational Chemistry: Theories and Models, $2^{\text {nd }}$ ed.; John Wiley: New York, 2004.

16. SPARTAN 08, Wavefunction Inc., Irvine, CA, 2000.

17. Arora, A.; Hydrocarbons, Discovery Publishing House: New Delhi, 2006.

18. Mariano, A.; Ventura, E.; do Monte, S. A.; Braga, C. F.; Carvalho. A. B.; Araújo, R.; Santana, O. L.; Quim. Nova 2008, 31, 1243.

19. Tüysüz, C.; IOJES 2010, 2, 37.

20. Taber, K. S.; Chem. Educ. Res. Pract. 2001, 2, 123.

21. Molina, M. F.; Carriazo, J. G.; Farías, D.; Quim. Nova 2011, 34, 1672.

22. Aksela, M. J.; Educació Química EduQ. 2011, 9, 30. 